\title{
Dielectric Characterization of composite building materials depending on the volume fraction for Mobile Phone Frequencies.
}

\author{
Caractérisation diélectrique de matériaux composites de \\ construction en fonction de la fraction volumique pour des \\ fréquences de téléphone mobile.
}

\author{
Jaouad Terhzaz ${ }^{1,2}$, Hassan Elmajid ${ }^{2}$ and Hassan Ammor ${ }^{2}$ \\ ${ }^{1}$ Centre régional des métiers de l'éducation et de la formation, Casablanca, Maroc \\ ${ }^{2}$ Université Mohammed V-Agdal, EMI, Lab. d'Electronique et de Communications, Rabat, Maroc
}

\begin{abstract}
This $\mathrm{W}$ presents a new technique of Dielectric Characterization of composites building materials. This technique is based on the modeling of an open coaxial cell with a mathematical formulation that links the admittances of the filled and empty cell to the complex permittivity $\varepsilon^{*}$ of materials characterization. We applied this technique to the dielectric characterization of some building materials powder in the frequency band $(100 \mathrm{MHz}-4 \mathrm{GHz})$. We also characterize some composite materials (sand- brick, cement- sand, and cement-brick) depending on the volume fraction for Mobile Phone Frequencies. We made a comparison with the laws of mixtures to identify the most appropriate law to render the dielectric behavior of these materials.
\end{abstract}

\begin{abstract}
Résumé. Ce travail présente une nouvelle technique de caractérisation diélectrique des matériaux de constructions composites. Cette technique est basée sur la modélisation d'une cellule coaxiale ouverte avec une formulation mathématique qui relie les admittances de la cellule remplie et vide à la permittivité complexe $\varepsilon^{*}$ des matériaux à caractériser. Nous avons appliqué cette technique à la caractérisation diélectrique de quelques matériaux de constructions du bâtiment en poudre dans la bande de fréquence $(100 \mathrm{MHz}-4 \mathrm{GHz})$. Nous caractérisons aussi quelques matériaux composites (sable-brique, ciment-sable, ciment-brique) en fonction de la fraction volumique pour des fréquences de téléphone mobile. Nous avons établi une comparaison avec les lois de mélanges afin d'identifier la loi la plus adaptée pour traduire le comportement diélectrique de ces matériaux.
\end{abstract}

\section{Introduction}

Dans l'étude de l'interaction du rayonnement électromagnétique avec la matière, les méthodes de mesure de l'évolution de la permittivité diélectrique complexe dans le domaine fréquentiel constituent un outil très important pour la caractérisation diélectrique des matériaux composites constituant les milieux de propagation. Par exemple, l'optimisation des performances des systèmes de communications sans fil utilisés à l'intérieur et à l'extérieur des bâtiments nécessite la caractérisation 
de canaux de propagation. La mise au point des modèles de propagation nécessite la connaissance de la permittivité diélectrique des matériaux composites de constructions [1-2]. Il existe plusieurs méthodes de détermination de la permittivité diélectrique des matériaux, dont les plus importantes sont relatives aux travaux récents [2-3]. La méthode de mesure que nous utilisons dans ce travail est basée sur la modélisation d'une cellule coaxiale ouverte avec une formulation mathématique qui relie les admittances de la cellule remplie et vide à la permittivité complexe $\varepsilon^{*}$ des matériaux à caractériser. Dans cette formulation apparaît des coefficients qu'il faut déterminer par étalonnage. La détermination de la permittivité complexe de quelques produits étalons par cette méthode de mesure permet la validation de l'étalonnage effectué.

Dans ce travail, nous appliquons cette méthode en vue de caractériser quelques matériaux diélectriques de construction du bâtiment en poudre (brique, sable, ciment...) dans la bande de fréquence $(100 \mathrm{MHz}-4 \mathrm{GHz})$. Nous caractérisons aussi quelques matériaux composites (sable-brique, ciment-sable, ciment-brique) en fonction de la fraction volumique (varie entre 0.1 et 0.9 ) pour des fréquences de téléphone mobile et nous établissons une comparaison avec les lois de mélanges afin d'identifier la loi la plus adaptée pour traduire le comportement diélectrique de ces matériaux composites.

\section{Dispositif de mesure}

Les mesures des permittivités diélectriques des matériaux sont réalisées à l'aide du dispositif de mesure représenté sur la figure1. Ce dispositif est composé d'une cellule coaxiale ouverte en standard SMA $3.5 \mathrm{~mm}$ et d'un analyseur de réseaux vectoriel HP 8720C (50MHz-20GHz). Pour tenir compte des imperfections $\mathrm{du}$ dispositif de mesure, un étalonnage de l'analyseur de réseaux est effectué au préalable par trois impédances court-circuit, circuit ouvert et charge adaptée $50 \Omega$. La cellule de mesure est une portion de ligne coaxiale en circuit ouvert remplie de matériau à caractériser. Elle est connectée par l'intermédiaire d'un câble coaxial à l'analyseur de réseaux pour permettre la mesure du coefficient de réflexion dans le plan de référence (figure1).

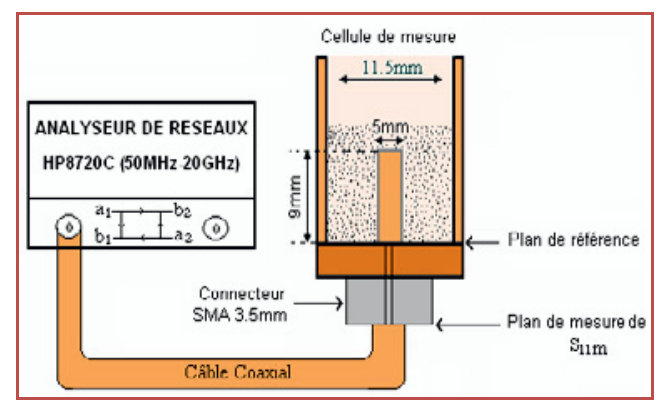

(a)

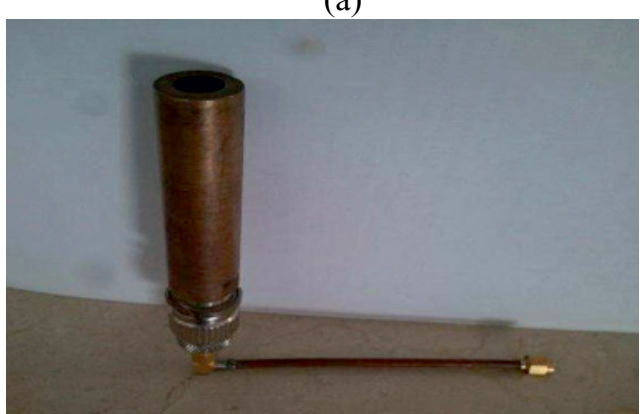

(b)

Fig. 1. Dispositif de mesure. (a) Schéma détail du dispositif de mesure, (b) Cellule coaxiale réalisée.

\section{Principe de la méthode}

La méthode de mesure de la permittivité diélectrique dans une bande de fréquence de $50 \mathrm{MHz}-4 \mathrm{GHz}$ est faite par la modélisation d'une cellule coaxiale type Bussey. Cette méthode consiste à relier l'admittance d'une cellule de mesure contenant un matériau de permittivité complexe $\varepsilon^{*}$ à la variable $S=\varepsilon^{*}-1$ par une fonction rationnelle à coefficient réels [4]. Pour la cellule utilisée, on se limite au modèle à deux paramètres $A$ et $B$ caractérisant la distribution du champ électrique dans celle-ci. A et $B$ sont déterminés expérimentalement par étalonnage de la cellule de mesure avec des produits étalons [5]. En utilisant l'équation générale de dispersion :

$$
\varepsilon^{*}=\varepsilon_{\infty}+\frac{\varepsilon_{o}-\varepsilon_{\infty}}{1+(j \omega \tau)^{1-\alpha}}=\varepsilon^{\prime}-j \varepsilon^{\prime \prime}
$$

Dont les valeurs de $\left(\varepsilon_{\infty}\right),\left(\varepsilon_{o}\right),(\tau)$ et $(\alpha)$ sont données dans le tableau ci-dessous [6] :

Tableau 1. Caractéristiques des produits étalons à $\mathrm{t}=25^{\circ} \mathrm{C}$

\begin{tabular}{|l|c|c|l|l|}
\hline Produits étalons & $\varepsilon_{0}$ & $\varepsilon_{\infty}$ & $\alpha$ & $\lambda(\mathrm{cm})$ \\
\hline Chlorobenzène & 2.40 & 5.63 & 0.04 & 1.94 \\
Chloroforme & 2.03 & 4.71 & 0 & 1.4 \\
Dichloro-1,2- & 10.16 & 2.35 & 0.01 & 1.31 \\
éthane & & & & 1.01 \\
Hexane & 2.17 & 1.89 & 0 & \\
\hline
\end{tabular}

L'équation de base est la suivante :

$\frac{\mathrm{Y}_{e}-\mathrm{Y}_{e o}}{\mathrm{Y}_{e o}}=\frac{\mathrm{A}\left(\varepsilon^{*}-1\right)}{1+\mathrm{B}\left(\varepsilon^{*}-1\right)}=\mathrm{Y}^{\prime}+j \mathrm{Y}^{\prime \prime}$

Où $\mathrm{Y}_{e}$ et $\mathrm{Y}_{e o}$ sont respectivement l'admittance de la cellule coaxiale remplie et vide.

La séparation de la partie réelle et imaginaire de l'équation (2) conduit à un système à deux équations : 


$$
\begin{aligned}
& \frac{\mathrm{Y}^{\prime \prime}}{\mathrm{Y}^{\prime}\left(\varepsilon^{\prime}-1\right)+\mathrm{Y}^{\prime \prime} \varepsilon^{\prime \prime}}=\frac{\mathrm{A}\left(\varepsilon^{\prime}-1\right)}{\mathrm{Y}^{\prime}\left(\varepsilon^{\prime}-1\right)+\mathrm{Y}^{\prime \prime} \varepsilon^{\prime \prime}}-\mathrm{B} \\
& \frac{\mathrm{Y}^{\prime \prime}}{\mathrm{Y}^{\prime} \varepsilon^{\prime \prime}-\mathrm{Y}^{\prime \prime}\left(\varepsilon^{\prime}-1\right)}=\frac{-\mathrm{A} \varepsilon^{\prime \prime}}{\mathrm{Y}^{\prime} \varepsilon^{\prime \prime}-\mathrm{Y}^{\prime \prime}\left(\varepsilon^{\prime}-1\right)}-\mathrm{B}
\end{aligned}
$$

D'après les résultats obtenus [4-5], l'utilisation des deux équations aboutit à des résultats imprécis. Par contre, l'utilisation de l'équation (3) écrite sous la forme de la régression linéaire $\mathrm{y}=\mathrm{Ax}+\mathrm{B}$, a permis d'obtenir des résultats meilleurs. Le calcul de A et de $\mathrm{B}$ consiste donc à considérer $\mathrm{n}$ produits étalons et pour chaque produit correspond un couple (xi, yi). Par application de la méthode des moindres carrée, les coefficients A et B sont déterminés [5]. Pour chaque fréquence, les coefficients $\mathrm{A}$ et B sont calculés à partir de l'étalonnage de la cellule, la permittivité diélectrique d'un produit quelconque est déduite de l'équation (2) dont le développement donne :

$$
\left(\varepsilon^{*}-1\right)\left(\mathrm{BY}^{\prime}+j \mathrm{~B} \mathrm{Y}^{\prime \prime}-\mathrm{A}\right)+\mathrm{Y}^{\prime}+j \mathrm{Y}^{\prime \prime}=0
$$

A partir de cette équation, on obtient :

$$
\begin{aligned}
& \varepsilon^{\prime}=1+\frac{\mathrm{Y}^{\prime}\left(\mathrm{A}-\mathrm{B} \mathrm{Y}^{\prime}\right)-\mathrm{B} \mathrm{Y}^{\prime \prime}}{\left(\mathrm{B} \mathrm{Y}^{\prime}-\mathrm{A}\right)^{2}+\left(\mathrm{B} \mathrm{Y}^{\prime \prime}\right)^{2}} \\
& \varepsilon^{\prime \prime}=-\frac{\mathrm{A} \mathrm{Y}^{\prime \prime}}{\left(\mathrm{B} \mathrm{Y}^{\prime}-\mathrm{A}\right)^{2}+\left(\mathrm{B} \mathrm{Y}^{\prime \prime}\right)^{2}}
\end{aligned}
$$

L'utilisation de ces deux équations pour chaque fréquence permet de calculer $\varepsilon^{\prime}$ et $\varepsilon^{\prime \prime}$ d'un matériau à partir des valeurs de $\mathrm{Y}^{\prime}, \mathrm{Y}^{\prime \prime}$, A et $\mathrm{B}$. L'incertitude sur les mesures de $\varepsilon^{\prime}$ et de $\varepsilon^{\prime \prime}$ sont respectivement de l'ordre de $4 \%$ et de $6 \%$. Cette façon de faire les mesures conduit évidemment à un programme de calcul simple. Beaucoup plus simple que les méthodes qui sont programmées par les constructeurs d'analyseur de réseaux pour calculer la permittivité d'un échantillon plaqué devant une cellule coaxiale ouverte notamment.

\section{Préparation des échantillons de matériaux de constructions}

Les matériaux de constructions étudiés sont des solides en poudre (brique rouge, ciment, sable côtier et le béton), préparés au sein de notre laboratoire. La brique rouge et le béton ont été broyés et tamisés pour que leur granulométrie soit très faible, en vue d'éviter les espaces libres entre les grains au cours de la mesure. Le sable côtier a été lavé à l'eau distillée puis séché pour éliminer les cristaux de sel qui proviennent de la salinité des eaux de mer. Le fait que nous désirons caractériser aussi quelques matériaux composites (sable-brique, ciment-sable, ciment-brique) en fonction de la fraction volumique et non seulement en fonction de la fréquence. Il est nécessaire de faire une étude préalable afin de déterminer la densité des matériaux et préparés les échantillons du mélange en fonction de la fraction volumique.

\section{Résultats et discussions}

Les séries de mesures sont effectuées en relevant les évolutions de la permittivité complexe $\left(\varepsilon^{\prime}, \varepsilon^{\prime \prime}\right)$ des échantillons en poudre de quelques matériaux utilisés dans la construction du bâtiment dans un large domaine de fréquences. Les résultats obtenus sont illustrés sur la figure 2 . On constate à partir de cette figure que les matériaux étudiés présentent de faibles pertes diélectriques $\left(\varepsilon^{\prime \prime} \leq 0.2\right)$. De plus, la permittivité diélectrique de ces matériaux reste presque constante lorsque la fréquence est supérieure à $200 \mathrm{MHz}$, elle est d'ordre de 3 pour le sable et la brique rouge. Pour le ciment et le béton la valeur de $\varepsilon^{\prime}$ est autour de 3,6. Des résultats intéressants montrent que ce type de matériaux suit le comportement de Debye avec conduction [7], ceci explique les valeurs de la permittivité diélectrique élevées à basse fréquence ( $\mathrm{f} \leq 200 \mathrm{MHz}$ ). Nous présentons dans la figure 3 les variations de la permittivité diélectrique mesurée, par la méthode développée dans ce travail, des deux matériaux composites Brique-Sable (B-S) et Ciment-Brique (C-B) en fonction de la fraction volumique $\Phi$ pour des fréquences de téléphone mobile $900 \mathrm{MHz}$ et $1.8 \mathrm{GHz}$. Les résultats obtenus montrent que ces deux matériaux composites présentent de faibles pertes diélectriques. De plus, la permittivité diélectrique reste presque constante pour le BriqueSable (d'ordre de 3.1) et décroît de 3.6 à 3.1 pour le
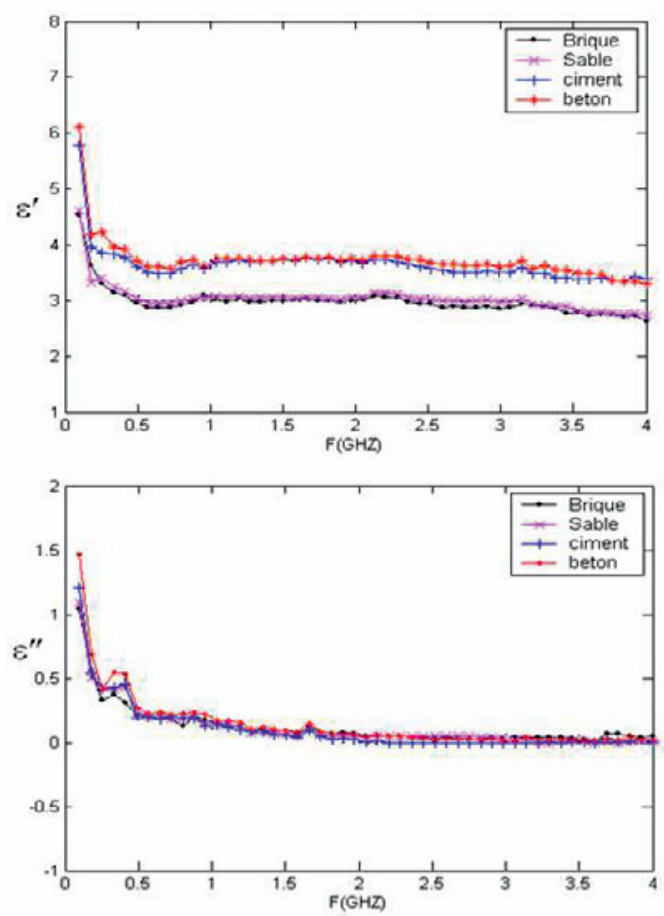

Fig. 2. La permittivité diélectrique de quelques matériaux de construction en fonction de la fréquence. 

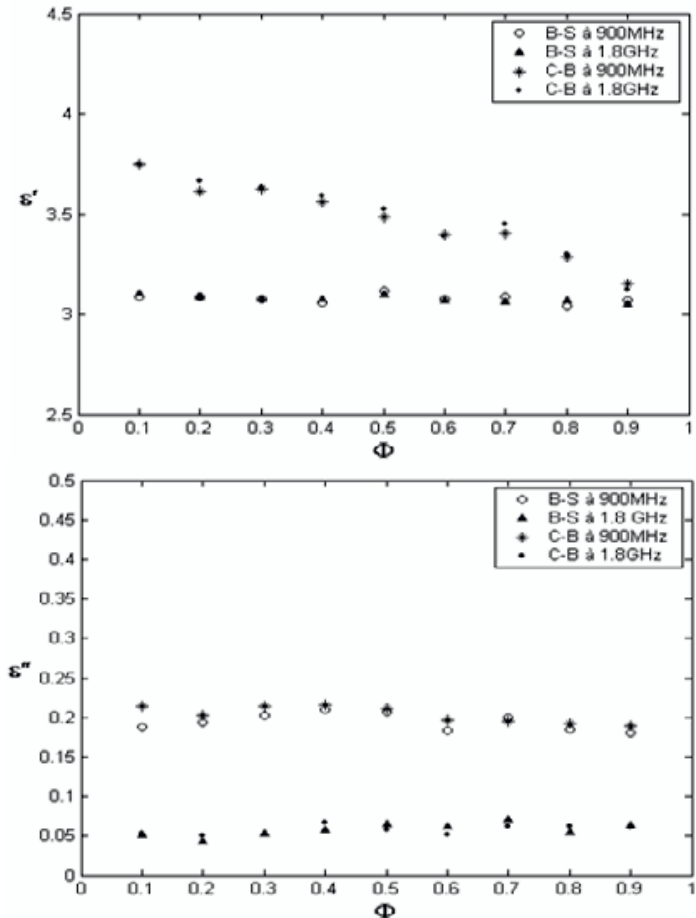

Fig. 3. Les variations de la permittivité diélectrique des deux matériaux composites B-S et C-B en fonction de la fraction volumique à $900 \mathrm{MHz}$ et à $1.8 \mathrm{GHz}$.

Ciment-Brique lorsque $\Phi$ passe de 0.1 à 0.9 . Pour interpréter ces résultats expérimentaux, nous avons comparé le comportement diélectrique des deux matériaux composites en utilisant les deux modèles de Lichtnecker et de Loyenga.
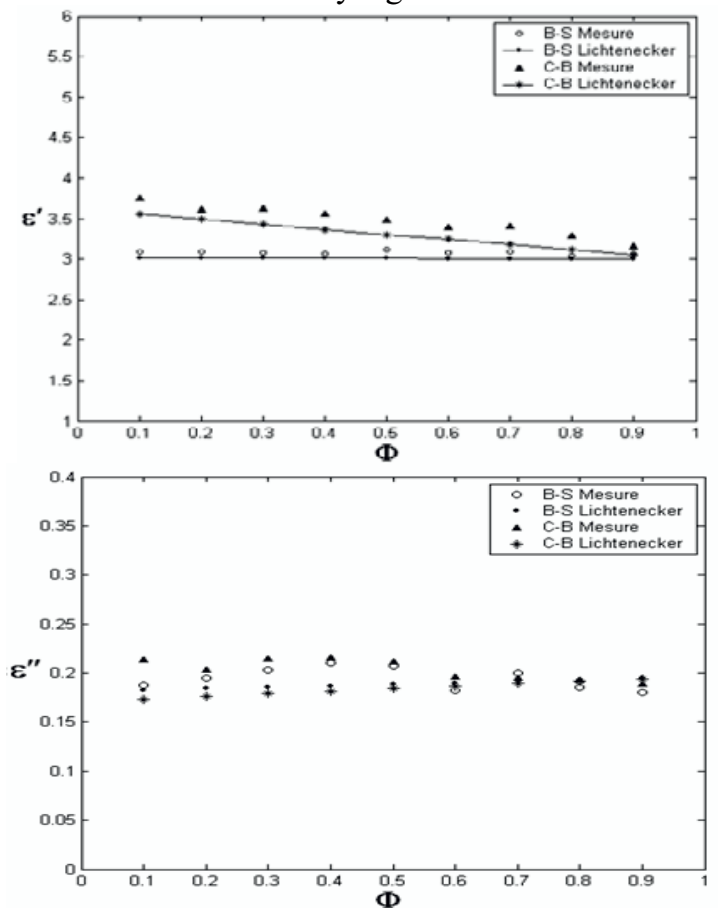

Fig. 4. Comparaison entre les mesures et la loi de mélange Lichtnecker pour les deux matériaux B-S et $\mathrm{C}-\mathrm{B}$ à $900 \mathrm{MHz}$.
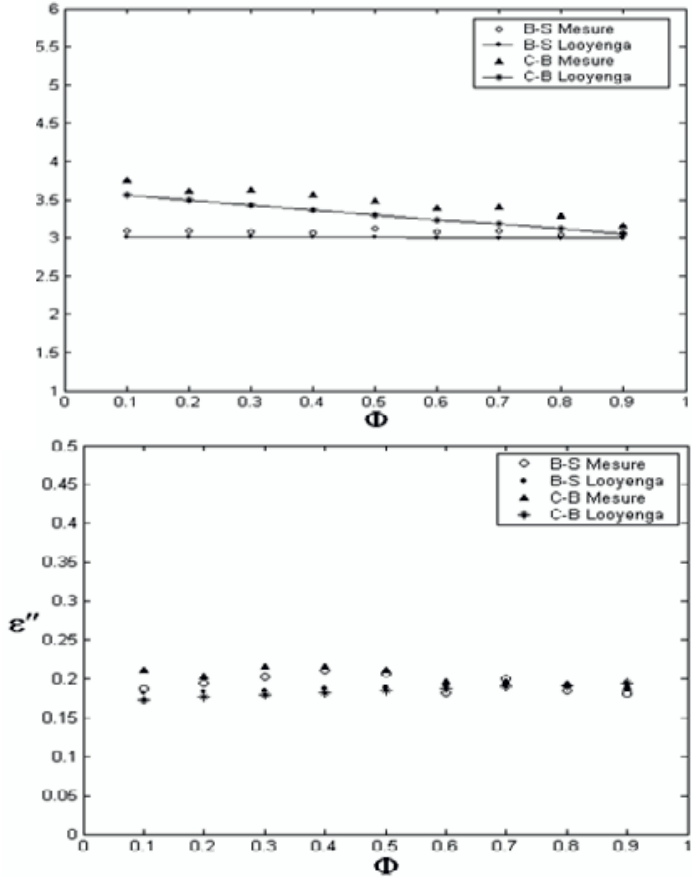

Fig. 5. Comparaison entre les mesures et la loi de mélange Loyenga pour les deux matériaux B-S et C-B à $900 \mathrm{MHz}$.

Les formules directes de ces deux modèles se présentent comme suit :

Modèle Lichtnecker : $\quad \varepsilon^{*}=\left(\varepsilon_{1}^{*}\right)^{\Phi}\left(\varepsilon_{2}^{*}\right)^{1-\Phi}$

Modèle Loyenga : $\left(\varepsilon^{*}\right)^{1 / 3}=\Phi\left(\varepsilon_{1}^{*}\right)^{1 / 3}+(1-\Phi)\left(\varepsilon_{2}^{*}\right)^{1 / 3}$

Avec $\Phi=V_{1} /\left(V_{1}+V_{2}\right)$ où $V_{1}$ et $V_{2}$ sont respectivement les volumes des milieux de permittivités $\varepsilon_{1}$ et $\varepsilon_{2}$.

L'étude comparative en fonction de la fraction volumique $\Phi$ est présentée sur les figures 4 et 5 pour la fréquence $900 \mathrm{MHz}$. Nous remarquons que les courbes de mesure et de simulation sont proches avec un écart d'ordre de 0.1. Ceci montre que le mélange binaire des matériaux de construction que nous avons étudié obéissant aux deux lois de Lichtnecker et de Loyenga.

\section{Conclusion}

Le dispositif de mesure réalisé dans ce travail permet de caractériser rapidement des matériaux de construction sous forme de poudre. Les résultats obtenus montrent que les matériaux composites de constructions étudiées présentent de faibles pertes diélectriques dans la bande de fréquence $100 \mathrm{MHz}-$ $4 \mathrm{GHz}$. Ceci a une conséquence directe sur des applications en télécommunications et surtout sur la propagation des ondes électromagnétiques dans ces matériaux. De plus, ces matériaux composites obéissent de manière assez satisfaisante les lois de mélanges de Lichtnecker et de Loyenga. 


\section{Références}

1. M. N.Afsar, J.R.Birch et R.N. Clarke:" The measurements of the properties of materials" IEEE-Procedings, Vol 74, $\mathrm{N}^{\circ} 1$, pp 183-199, (1986).

2. F. Sagnard, C. Vignat, V. Moncourtois, E. Rolland: "Détermination de la permittivité complexe de matériaux de construction dans le domaine micro-onde: Comparaison des méthodes de Fresnel et d'Ellipsométrie" $2^{\text {ème }}$ Journées nationales micro-ondes, Poitiers16-1718, pp 5c-1, Mai, (2001).

3. J. J. Bonte : " Contribution à la caractérisation électromagnétique de nouveaux matériaux dans une large gamme de fréquence. Doctorat de l'université de Lille, $\mathrm{N}^{\circ}$ d'ordre 549, France, Juin (1990).

4. H. Ammor, J. M. Thiebaut, G. Roussy: " Etalonnage des cellules de mesure de la permittivité diélectrique dans un large domaine de fréquence" Onde Electrique, Vol 74, $\mathrm{N}^{\circ} 4$, Juillet-Août ,(1994).

5. H. E. Bussey :" Dielectric measurements in a shielded open circuit coaxial line" IEEE trans. Instrum. Meas, Vol-IM29, pp 120, (1980).

6. NBS Circular 514: " Table of dielectric constants of pure liquids " United states departement of commerce (National Bureau of standard).

7. Tarik Zakaria: "Contribution à l'étude des propriétés diélectrique de matériaux poreux en vue de l'estimation de leur teneur en eau" Thèse de Doctorat, Institut National Polytechnique de Grenoble, France, Octobre, (1997). 